\title{
Infecção pelo HIV/Aids em população indígena: estudo transversal
}

\author{
HIV/AIDS infection in indigenous population: a cross-sectional study \\ Infección por VIH/SIDA en población indígena: un estúdio transversal
}

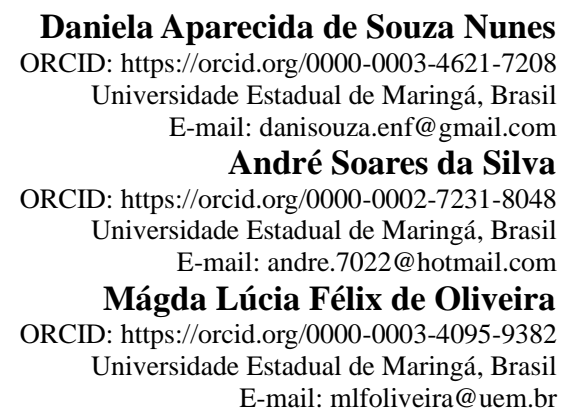

\begin{abstract}
Resumo
Objetivo: Descrever aspectos epidemiológicos e a evolução da infecção pelo HIV entre povos indígenas da região sul do estado do Mato Grosso Sul. Método: Estudo transversal, descritivo e retrospectivo, realizado a partir de registros do Sistema de Informação de Agravos de Notificação dos anos 2005-2018. Dados de indivíduos com 13 anos ou mais, da população indígena assistida pelo Distrito Sanitário Especial Indígena do Polo Base de Japorã e diagnóstico de infecção pelo HIV/Aids, foram transferidos para uma planilha de comparação com sistema de informação local. Para o cálculo da prevalência e evolução de casos foi utilizado o número de casos novos anual de HIV/Aids. Resultados: Foram identificados $17(65,4 \%)$ casos em mulheres, 13 (72,2\%) entre 20-39 anos. Cinco (19,2\%) evoluíram para Aids. As taxas de detecção do HIV/Aids, para os anos de 2016 e 2018 foram de 115.54 e 155.49 respectivamente. As taxas de prevalência do HIV apresentaram flutuações entre os anos 2016, 217 e 218 . A aldeia Porto Lindo apresentou uma taxa de detecção de 59,878/100 mil habitantes, Yvykatu 400,802/100 mil habitantes e a Pyelito Kuê 602,409/100 mil habitantes. A taxa de óbito por Aids na aldeia Porto Lindo foi de 2,24/10 mil habitantes e no Yvykatu 40,08/10 mil habitantes. A taxa de abandono para a aldeia Porto Lindo foi de 6,73/10 mil habitantes. Conclusão: As taxas de deteç̧ão de HIV apresentaram tendência de crescimento no povo indígena estudado. É necessário que sejam realizados estudos para direcionamento de ações que impactem os indicadores de prevalência da infecção.
\end{abstract}

Palavras-chave: Infecções por HIV; Saúde de populações indígenas; Povos indígenas; Estudos transversais.

\begin{abstract}
Objective: To describe epidemiological aspects and the evolution of HIV infection among indigenous people in the southern region of the state of Mato Grosso do Sul. Method: Cross-sectional, descriptive and retrospective study, based on records from the Notifiable Diseases Information System in the years 2005 -2018. Data from individuals aged 13 years and over, from the indigenous population assisted by the Special Indigenous Health District of the Japorã Base Pole and diagnosis with HIV/AIDS infection, were transferred to a comparison spreadsheet with the local information system. To calculate the prevalence and evolution of cases, the annual number of new HIV/AIDS cases was used. Results: 17 (65.4\%) cases were identified in women, $13(72.2 \%)$ between 20-39 years. Five (19.2\%) developed AIDS. The HIV/AIDS detection rates for the years 2016 and 2018 were 115.54 and 155.49 respectively. HIV prevalence rates fluctuated between the years 2016, 217 and 218. The Porto Lindo village had a detection rate of 59.878/100 thousand inhabitants, Yvykatu 400.802/100 thousand inhabitants and Pyelito Kuê 602.409/100 thousand inhabitants. The AIDS death rate in the Porto Lindo village was 2.24/10 thousand inhabitants and 40.08/10 thousand inhabitants in Yvykatu. The abandonment rate for the Porto Lindo village was 6.73/10 thousand inhabitants. Conclusion: HIV detection rates tended to increase in the indigenous people studied. It is necessary that studies be carried out to direct actions that impact the infection prevalence indicators.
\end{abstract}

Keywords: HIV infections; Health of indigenous peoples; Indian people; Cross-sectional studies.

\section{Resumen}

Objetivo: describir los aspectos epidemiológicos y la evolución de la infección por el VIH entre los pueblos indígenas de la región sur del estado de Mato Grosso do Sul. Método: estudio transversal, descriptivo y retrospectivo, basado en los registros del Sistema de Información de Enfermedades de Declaración Obligatoria en los años 2005 -2018. Los 
datos de personas con 13 años o más, de la población indígena atendida por el Distrito Especial de Salud Indígena del Polo Base Japorã y diagnosticadas con infección por VIH/SIDA, fueron transferidos a una ficha de comparación con el sistema de información local. Para el cálculo de la prevalencia y evolución de los casos se utilizó el número anual de nuevos casos de VIH/SIDA. Resultados: se identificaron 17 (65,4\%) casos en mujeres, 13 (72,2\%) entre 20-39 años. Cinco $(19,2 \%)$ desarrollaron SIDA. Las tasas de detección de VIH/SIDA para los años 2016 y 2018 fueron 115,54 y 155,49 respectivamente. Las tasas de prevalencia del VIH fluctuaron entre los años 2016, 217 y 218 . La aldea Porto Lindo tuvo una tasa de detección de 59.878/100 mil habitantes, Yvykatu 400.802/100 mil habitantes y Pyelito Kuê 602.409/100 mil habitantes. La tasa de mortalidad por SIDA en la aldea Porto Lindo fue de 2,24/10 mil habitantes y de 40,08/10 mil habitantes en Yvykatu. La tasa de abandono para la aldea Porto Lindo fue de 6,73/10 mil habitantes. Conclusión: Las tasas de detección del VIH tendieron a aumentar en los indígenas estudiados. Es necesario que se realicen estudios para encaminar acciones que impacten en los indicadores de prevalencia de contagios.

Palabras clave: Infecciones por VIH; Salud de los pueblos indígenas; Gente India; Estudios transversales.

\section{Introdução}

A complexidade e o caráter dinâmico da infecção pelo HIV/Aids e a marca da promiscuidade, da culpa e da morte, produziram, desde o início da epidemia, mudanças epidemiológicas que tiveram importantes respostas sociais e políticas (Brasil, 2021). A Infecção pelo HIV/Aids revelou-se, mundialmente, como grave problema de saúde pública em decorrência de suas altas taxas de morbimortalidade. No Brasil, a epidemia se propagou nas diferentes regiões do país, assumindo características epidemiológicas específicas em diversos grupos populacionais (Orellana et al., 2013; Santos, 2016; Silva et al., 2018; Woszniak et al., 2020).

Até dezembro de 2019, 38 milhões de pessoas viviam com o HIV mundialmente, a maioria em países pobres ou em desenvolvimento, e aproximadamente 7,1 milhões não sabiam que eram portadores do HIV. Contudo, desde 1998 o número anual de novas infecções diminuiu cerca de 40\%, passando de 2,8 milhões para 1,7 milhões em 2019, representando um declínio substancial, embora heterogêneo entre países e grupos populacionais (Unaids, 2021).

No Brasil, o primeiro caso diagnosticado de infecção pelo HIV ocorreu em 1980, no município de São Paulo e o Brasil é também o país que mais concentra casos de novas infecções na América Latina, respondendo por $40 \%$ das novas infecções (Dartora, et al., 2017). No entanto, a notificação de infecção pelo HIV passou a compor a lista do Sistema de Informação de Notificação de Agravos - SINAN em junho de 2014. Em período anterior apenas os casos de aids e de infecção pelo HIV em gestante, parturiente, puérpera e criança exposta ao risco de transmissão vertical eram notificados (Brasil, 2014).

No período de 1980 a junho de 2020, foram detectados 1.011.617 casos de aids no Brasil. A análise dos dados de 2009 apontou um declínio na taxa de detecção da aids de 17,2\%, passando de 21,5 em 2009 para 17,8/100 mil habitantes em 2019. Porém, essa tendência não ocorreu em todo território nacional: na Região Centro-oeste a epidemia apresentou um aumento de 2,7\% na taxa de detecção nos últimos 10 anos, passando de 18,6 em 2009 para 19,1/100 mil habitantes em 2019 (Brasil, 2020).

A epidemia de aids no Brasil se distribui associada aos determinantes sociais e de saúde, bem como ao acesso aos serviços de saúde, fatores sociodemográficos, econômicos e culturais, que determinam situação de maior ou menor vulnerabilidade de grupos populacionais aos riscos de infecção e disseminação do vírus (Granjeiro, et al., 2010; Graeff et al., 2021).

Esta situação reflete um quadro de ocorrência da infecção pelo HIV em seguimentos populacionais em condições de maior vulnerabilidade (Ribeiro et al., 2020), que não estão adequadamente alcançadas por medidas de prevenção, tratamento e cuidado de forma integral, em função da situação de exclusão e dificuldades de acesso aos serviços de saúde (Pereira et al., 2019).

Os povos indígenas, ao longo da história, têm sido particularmente impactados por epidemias de doenças infecciosas, estendendo-se com efeitos profundos e duradouros a todos os âmbitos da vida desses. Em comparação à população não indígena em geral ocorrem elevada prevalência de carências nutricionais, doenças crônicas não transmissíveis e doenças 
infecto-parasitárias (Ministério da Saúde, 2020; Polanco-Pasage et. al., 2021). O colonialismo, racismo, exclusão social e dificuldades de acesso a serviços de saúde foram identificados como determinantes que aumentam a vulnerabilidade das populações indígenas ao HIV (Teixeira, 2014; Negin et al., 2015), refletida em taxas elevadas de detecção da infecção entre os indígenas (Negin et al., 2015).

No Brasil, ainda não há informações suficientes para estimar a dimensão da infecção pelo HIV/Aids entre os povos indígenas. Estudos evidenciam condições de vulnerabilidade, onde são observados níveis de escolaridade baixo, condições socioeconômica desfavoráveis, além de uma exclusão social histórica, como fatores de risco (Negin et al., 2015; Santos et al., 2019). Outros apontam altas prevalências de infecções sexualmente transmissíveis e expansão de casos de infecção pelo HIV em áreas indígenas de fronteira e próximas aos centros urbanos (Benzaken et al., 2017; Graeff et al., 2019).

O primeiro caso de aids em indígenas foi registrado em 1988 na região Sul do país, mas somente em 1998 foi iniciado o primeiro estudo de investigação sistemática da infecção em território indígena. Neste primeiro estudo, com notificação de 33 casos de aids, foram considerados como importantes fatores para a disseminação a mudança no perfil epidemiológico da doença, caracterizado pela pauperização e interiorização da infecção, e a ausência de uma política de saúde específica, considerando a precariedade da atenção à saúde indígena neste cenário (Brasil, 2000; Negin et al., 2015; Graeff et al., 2019).

Estima-se no Brasil, a existência de 896.000 indígenas, distribuídos em 305 etnias (IBGE, 2012; Nobrega et al., 2020). O Estado do Mato Grosso do Sul representa a segunda maior população indígena do Brasil. O Distrito Sanitário Especial Indígena do Mato Grosso do Sul (DSEI-MS), que integra o Subsistema de Atenção à Saúde Indígena (SASI) do Sistema Único de Saúde (SUS), informou uma população de 83.241 indígenas no ano de 2017. Essas populações estão distribuídas em oito etnias - Kaiowá, Guarani- nhandéva, Terena, Kadiwéu, Kinikinau, Guató, Ofaié e Atikun -, entre aldeias e acampamentos de 31 municípios do estado (Brasil, 2017).

Considerando a magnitude da infecção pelo HIV em seguimentos populacionais vulneráveis e a prevalência do HIV/Aids como realidade entre populações indígenas (Graeff et al., 2019), mas que não existem informações suficientes para estimar a dimensão da infecção pelo HIV, a presente análise possui relevância, pois trata de um estudo que permite conhecer os aspectos relacionados à infecção do HIV entre a população indígena de um polo base da região sul do Mato Grosso Sul onde estão concentrados $68 \%$ da população indígena do estado.

Assim, a partir da lacuna relacionada ao entendimento da dinâmica da infecção nestes povos, este estudo teve como objetivo descrever aspectos epidemiológico e a evolução da infecção pelo HIV entre povos indígenas da região sul do estado do Mato Grosso Sul.

\section{Metodologia}

Estudo descritivo, transversal e retrospectivo, que considerou o território indígena situado no Polo Base de Japorã do estado de Mato Grosso do Sul. Os estudos descritivos compreendem descrição detalhada e organizada do fenômeno estudado estabelecendo correlação entre as variáveis que definem sua natureza (Zangirolami-Raimundo et al., 2018). O estudo transversal é definido como pesquisas que demonstram a situação de saúde de uma população. Objetiva, além da descrição dos fenômenos, conhecer a relação de causa e efeito, analisando as relações entre os fatores de risco e fatores determinantes (Zangirolami-Raimundo et al., 2018).

A população indígena deste Polo, de predominância da etnia Guarani-nhandéva, é constituída por 5.788 indígenas distribuídos em duas aldeias e três acampamentos, pertencentes ao Distrito Sanitário Especial Indígena de Mato Grosso do Sul - DSEI-MS e localizados em quatro municípios do estado do Mato Grosso do Sul - Iguatemi, Japorã, Eldorado e Sete Quedas, (IBGE, 2012; Brasil, 2017). 
A Figura 1 mostra a distância dos territórios indígenas com relação à estrutura administrativa do Polo Base, localizado até o ano de 2018, no município de Iguatemi. Atualmente essa estrutura está localizada na aldeia Porto Lindo - município de Japorã.

Figura 1: Mapeamento dos Territórios Indígenas e seus respectivos municípios. Polo Base de Japorã - Distrito Sanitário Especial Indígena do Mato Grosso do Sul- Brasil, 2018.

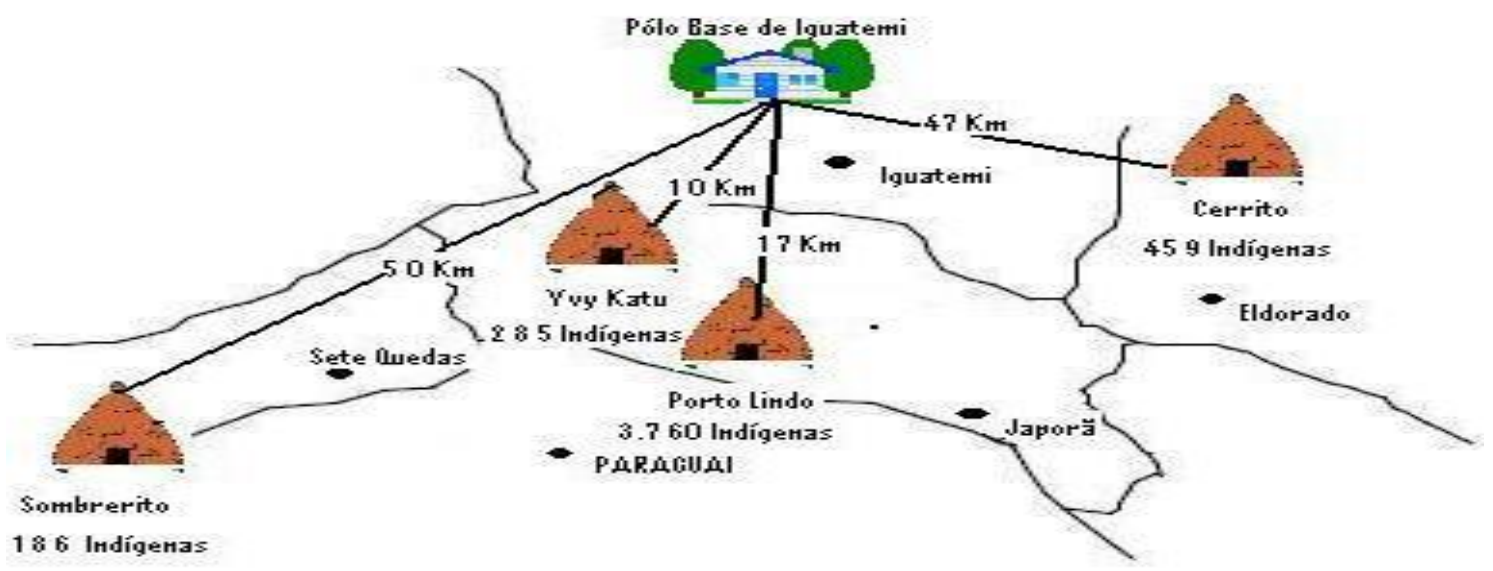

Fonte: DSEI-MS

Foram incluídos no estudo os indígenas com 13 anos ou mais assistidos pelo DSEI-MS, com diagnóstico de infecção pelo HIV, e residentes nas aldeias e acampamentos do Polo Base de Japorã no período do estudo. Foram excluídos os registros duplicados, não indígenas residentes nas aldeias ou acampamentos, indígenas residentes em áreas urbanas não assistida pelo Polo Base e crianças expostas ao HIV.

As informações sobre os casos de HIV/Aids foram obtidas a partir de duas fontes secundarias: o banco de dados do Sistema Nacional de Agravos de Notificação - SINAN dos municípios com populações indígenas pertencentes ao Polo Base de Japorã, e uma planilha de comparação de dados do SINAN com o Sistema de Informação da Saúde Indígena - SIASI. Estes registros apresentam dados de casos de infecção de HIV/Aids identificados no período, exceto a identificação do indivíduo, garantindo a confidencialidade das informações. As características sociodemográficas de idade, sexo, etnia e as bases populacionais para o cálculo das taxas foram obtidas no SIASI.

Foi utilizado o número de casos novos identificados em cada ano para cálculo da taxa de detecção dos casos de HIV na população estudada e, para o cálculo das taxas de prevalência foram considerados casos novos e antigos da infeção, excluindo-se os óbitos e transferências no período a análise descritiva foi realizada por medida de tendência central de média e de medidas de dispersão de variância e desvio padrão. Para a análise da prevalência da infecção na população foi utilizado o teste qui-quadrado, com um nível de significância de $5 \%$.

Não foram acessados dados nominais dos pacientes ou outros que possibilitassem sua identificação, visto que a pesquisa foi realizada a partir de banco de dados secundários. Dessa forma, não foi necessária a submissão ao Comitê de Ética em Pesquisa.

\section{Resultados}

No período do estudo, foram registrados 26 casos de infecção por HIV e aids. De acordo com a distribuição sociodemográfica dos casos, considerando sexo e faixa etária, foram identificados $17(65,4 \%)$ casos da infecção em mulheres 
indígena e nove (34,6\%) em homens. No entanto, entre os anos de 2005-2010 não foi identificado nenhum caso da doença por HIV/Aids na população masculina (Tabela 1).

Tabela 1. Distribuição dos casos de infecção pelo HIV/Aids, segundo sexo e faixa etária. Polo Base de Japorã - Distrito Sanitário Especial Indígena do Mato Grosso do Sul- Brasil, 2005 - 2018.

\begin{tabular}{ccc}
\hline Variável & N & \% \\
Sexo & & 34,6 \\
\hline Masculino & 9 & 65,4 \\
\hline Feminino & 17 & \\
\hline Faixa Etária & 1 & 3,8 \\
\hline $15-19$ & 6 & 23,1 \\
$20-29$ & 12 & 46,2 \\
$30-39$ & 3 & 11,5 \\
$40-49$ & 2 & 7,7 \\
$50-59$ & 2 & 7,7 \\
\hline 60 & $\mathbf{2 6}$ & $\mathbf{1 0 0 , 0}$ \\
\hline Total &
\end{tabular}

Fonte: Autores.

A faixa etária de maior detecção de infecção pelo HIV foi de 20-39 anos de idade, sendo identificados 18 casos nesta faixa etária $(69,23 \%)$, com predominância da população feminina com treze casos $(72,2 \%)$.

Do total de casos, cinco (19,2\%) evoluíram para aids no período em estudo, sendo a maioria do sexo masculino, quatro dos cinco casos notificados. Quanto ao tempo de evolução entre o diagnóstico de infecção pelo HIV e o "desenvolvimento da aids", três indivíduos foram diagnosticados com aids no momento do diagnóstico primário - dois homens e uma mulher.

A média de casos identificados foi de 6,5 casos/ano, com uma variação de um caso no ano de 2017 e nove casos no ano de 2018 e desvio padrão de 5,44. O ano de 2016 apresentou o menor desvio e os anos 2009 -2015 apresentaram o maior desvio padrão.

As taxas de detecção do HIV/Aids apresentaram flutuações anuais no período do estudo. Os anos de 2016 e 2018 registraram as maiores taxas, 115.54 e 155.49 respectivamente (Figura 2). 
Figura 2. Taxas anuais de detecção (por 100 mil habitantes) de casos de infecção pelo HIV/aids na população indígena atendida pelo Polo Base de Japorã - Distrito Sanitário Especial Indígena do Mato Grosso do Sul- Brasil, 2005 - 2018.

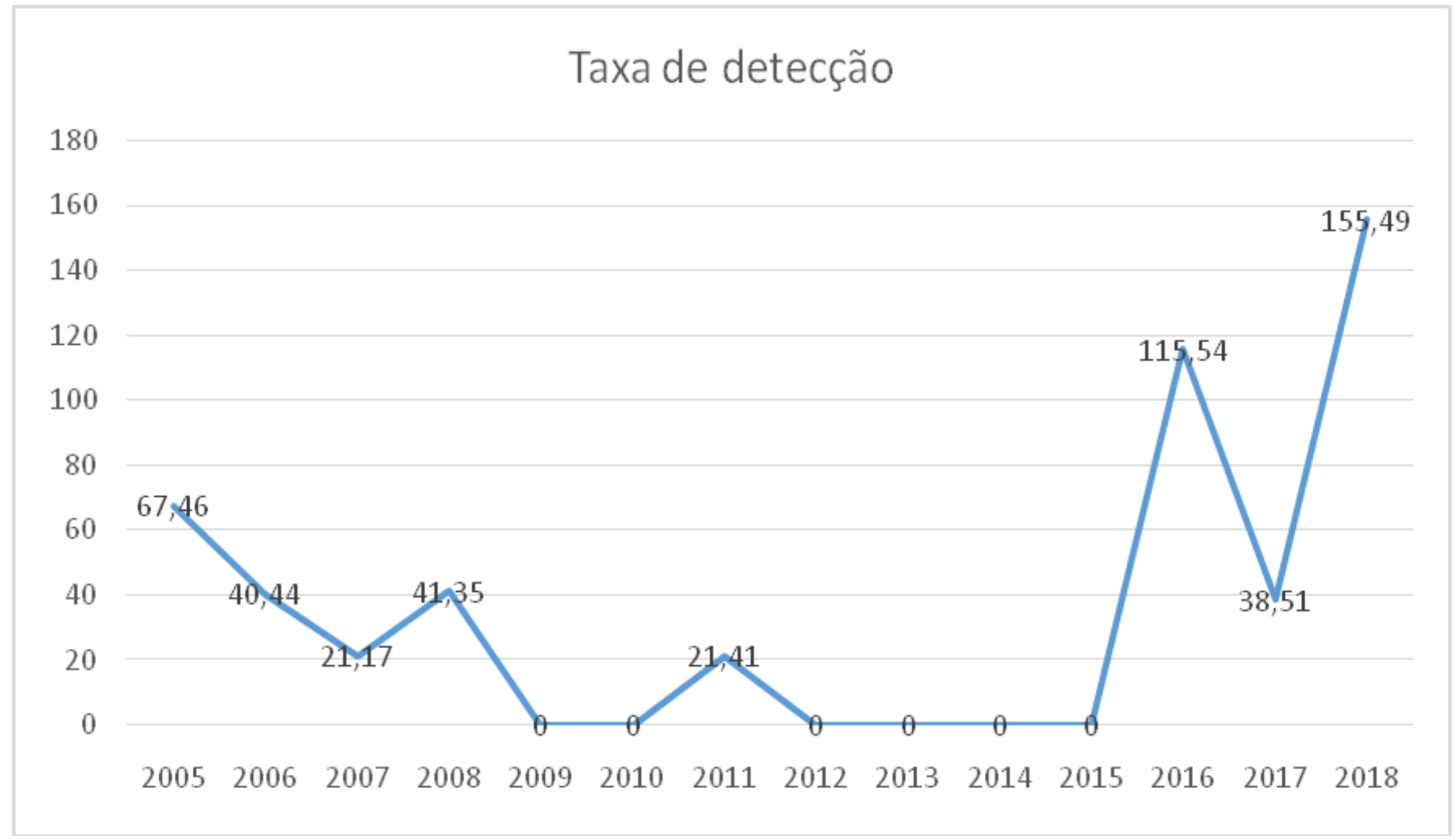

Fonte: Polo Base de Japorã/DSEI-MS

Os oito casos diagnosticados até o ano de 2008 foram em gestantes e em 2011 o Polo registrou o primeiro diagnóstico da infecção em homem.

No ano de 2016 foi observado mudança no perfil epidemiológico da infecção sendo identificados seis novos casos, três homens e três mulheres. Os casos identificados nos anos de 2017 e 2018 seguiram o mesmo padrão epidemiológico de 2016, sendo identificados cinco casos na população masculina e seis casos na população feminina.

Todos os casos identificados da infecção pelo HIV entre os anos de 2005 e 2008, foram na população feminina por meio de coleta de amostra de sangue em papel filtro, principal meio diagnóstico de agravos em gestantes ofertado pelos serviços de saúde, neste cenário.

As taxas de prevalência do HIV também apresentaram flutuações, observando-se uma ordem crescente entre os anos 2005 e 2012, diminuição nos anos seguintes e novamente um aumento entre os anos 2016, 217 e 218 (Figura 3). 
Figura 3. Taxas anuais (por 100 mil habitantes) de prevalência de casos de infecção pelo HIV/aids na população indígena atendida pelo Polo Base de Japorã - Distrito Sanitário Especial Indígena do Mato Grosso do Sul- Brasil, 2005 - 2018.

\section{Taxa de Prevalência}

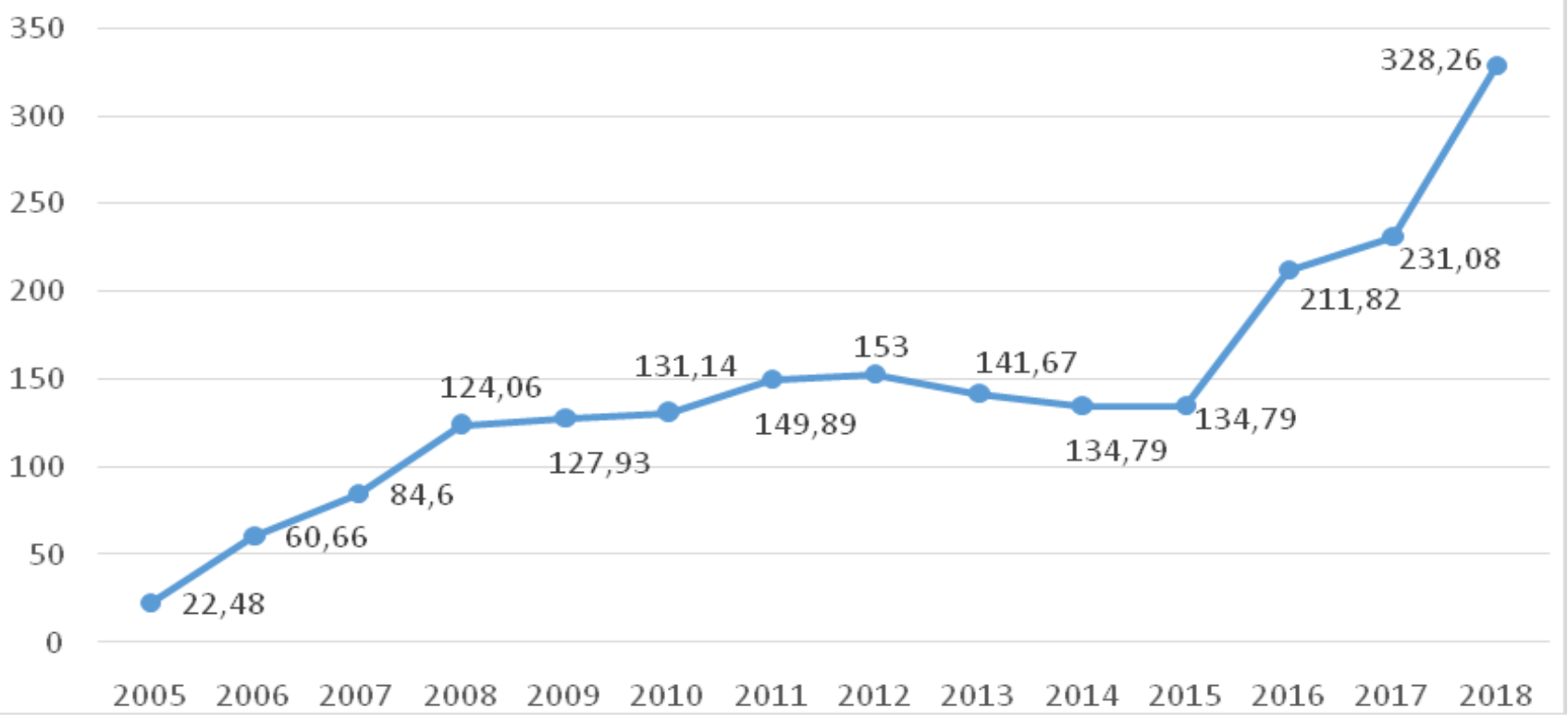

Fonte: Polo Base Japorã/DSEI-MS

A prevalência da infecção por HIV entre indígenas do Polo Base de Japorã passou de 22,48/100 mil em 2006 para 328,26/100 mil em 2018. Já a prevalência de aids passou de 20,22/100 mil em 2006 para 86,38/100 mil em 2018.

Dos cinco territórios (duas aldeias e três acampamentos), três registraram casos de infecção pelo HIV. A aldeia Porto Lindo concentrou a maioria, com 20 casos e uma taxa de detecção de 59,878/100 mil habitantes, as taxas de detecção dos acampamentos Yvykatu e Pyelito Kue foram 400,802/100 mil habitantes e 602,409/100 mil habitantes, porém é necessário considerar o contingente populacional desta última comunidade. (Tabela 2).

Tabela 2. Taxas de detecção de HIV/aids, de mortalidade e de abandono nas três aldeias identificadas com casos. Polo Base de Japorã - Distrito Sanitário Especial Indígena do Mato Grosso do Sul - Brasil, 2018.

\begin{tabular}{cccccccc}
\hline Aldeia & População & $\begin{array}{c}\text { HIV } \\
(\mathrm{n})\end{array}$ & $\begin{array}{l}\text { Taxa de detecção } \\
\text { de HIV (100 mil } \\
\text { hab.) }\end{array}$ & $\begin{array}{c}\text { Óbitos por } \\
\text { Aids (n) }\end{array}$ & $\begin{array}{c}\text { Taxa de } \\
\text { óbito (10 mil } \\
\text { hab.) }\end{array}$ & $\begin{array}{c}\text { Abandono (n) } \\
\text { Taxa de abandono } \\
(10 \text { mil hab.) }\end{array}$ \\
\hline Port.Lindo & 4.453 & 20 & 59,878 & 1 & 2,24 & 3 & - \\
Yvykatu & 499 & 4 & 400,802 & 2 & 40,08 & - & - \\
Pyelito K. & 166 & 2 & 602,409 & - & - & $\mathbf{5}$ & $\mathbf{5}$ \\
Polo Base & $\mathbf{5 . 1 1 8}$ & $\mathbf{2 6}$ & $\mathbf{6 2 , 6 7 4}$ & $\mathbf{3}$ & $\mathbf{5 6}$ & $\mathbf{3}$ \\
\hline
\end{tabular}

Fonte: Polo Base de Japorã/DSEI-MS.

Sobre as taxas de óbitos por aids, a aldeia Porto Lindo apresentou uma taxa de 2,24/10 mil habitantes (um óbito) e o Yvykatu uma taxa de 40,08/10 mil habitantes (dois óbitos). Para a aldeia Porto Lindo o período entre o diagnóstico e a ocorrência do óbito variou entre nove e 12 anos, e o terceiro óbito ocorreu no mesmo ano em que foi realizado o diagnóstico. 
Sobre os casos de abandono de tratamentos em serviços de saúde foi encontrada uma taxa de 6,73/10 mil habitantes para a aldeia Porto Lindo, dois na população masculina e um na população feminina.

\section{Discussão}

Devido ao processo gradativo de absorção dos casos de infecção pelo HIV pela rede de vigilância em saúde a partir de 2014 (Brasil, 2014), as taxas apresentadas no Boletim Epidemiológico de HIV/Aids do Ministério da Saúde são referentes aos casos de Aids e os casos de infecção pelo HIV são restritos aos números absolutos (Brasil, 2020).

No ano de 2005 foi identificado o primeiro caso de HIV no território, cujo diagnóstico foi realizado em outro polo base da região sul do estado de Mato Grosso do Sul. E ano foram notificados dois casos da infecção em gestantes. O estudo aponta a distribuição das taxas de infecção na população indígena do Polo Base de Japorã a partir desses casos.

A população com maior vulnerabilidade para a infecção do HIV está entre jovens e mulheres. É importante lembrar que as mulheres indígenas apresentam condições de saúde diferente da população feminina geral. São mulheres que experimentam a maternidade muito cedo, resultando em altas taxas de fecundidade (Coimbra, 2014). As altas taxas da infecção encontradas entre a população feminina na faixa etária de 20-39 anos contribuíram para uma alta taxa de prevalência da infecção neste grupo. Este achado reflete a necessidade de estratégias de enfrentamento do HIV nos territórios indígenas, considerando a organização das relações sociais entre homens e mulheres indígenas (Maúngue, 2020).

A predominância da população feminina nos casos identificados nos primeiros cinco anos do período não reflete resultados de estudos realizados anteriormente no estado de Mato Grosso do Sul, onde o maior número de casos foram encontrados na população masculina (Schimitt et al., 2018). Esta predominância feminina pode estar associada a dificuldade de acompanhamento dos homens nos serviços de saúde (Brasil, 2017; Trindade et al., 2021).

$\mathrm{O}$ resultado sugere a existência de desigualdades de gênero para as mulheres indígenas, apontando para maior vulnerabilidade ao HIV e outras Infecções Sexualmente Transmissíveis - ISTs. Essa situação de vulnerabilidade pode estar relacionada à forma como essas mulheres se relacionam com seus parceiros e os seus papéis na sociedade indígena (Abati, 2015), além de dificuldade de acesso aos serviços de saúde, baixa adesão ao uso de preservativos e ações de prevenção inadequadas que não compreendem às especificidades culturais indígenas (Portocarrero, 2015; Nobrega et al., 2020; Gomes et al., 2017).

No entanto não é possível assegurar que existe uma feminização da infecção nos territórios indígenas, uma vez que o acompanhamento da população masculina é marcado por dificuldades relacionadas a ausência desta população em períodos sazonais do ano por motivos de contrato de trabalho, que reflete na ausência nos serviços de saúde da aldeia e consequente baixa de cobertura diagnóstica nesta população (Graeff et al., 2019).

Para as mulheres indígenas na faixa etária sexualmente ativa, a testagem sorológica do HIV no pré-natal representa uma oportunidade de identificação oportuna da infecção (Abati, 2015). Nesse contexto, chama a atenção a maior possibilidade de transmissão vertical do HIV entre indígenas, considerando os aspectos culturais e as ações de educação e promoção da saúde, e de prevenção das ISTs se mostrarem ineficientes dentro dos territórios indígenas (Portocarrero, 2015; Tiago et al., 2017).

O aumento significativo nas taxas gerais de deteç̧ão nos anos de 2016 e 2018 pode estar associado ao aumento da capacidade de testagem rápida e ações específicas no ano de 2016, como a qualificação dos profissionais de saúde para a abordagem diagnóstica por meio dos testes rápidos (Graeff et al., 2019). Destaca-se que estratégias que favoreçam o aumento da cobertura diagnóstica precisam ser consideradas a partir do contexto cultural e social dos povos indígenas, considerando os conceitos de vulnerabilidades e fatores de risco para esta população (Maia et al, 2019). 
A identificação precoce com o início de tratamento constitui-se como uma das principais estratégias para o controle do progresso da infecção, sendo necessário a articulação entre gestores de saúde, responsáveis técnicos e equipes de saúde para favorecer as ações e garantia de acesso a esses meios diagnósticos (Lima, 2018).

$\mathrm{O}$ aumento da cobertura diagnóstica, a oferta da terapia antirretroviral na prestação de cuidados contínuos e a supressão viral em pessoas vivendo com HIV, em nível mundial, compõem a Meta 90-90-90. Essa proposta, do Programa Conjunto das Nações Unidas sobre HIV/Aids - UNAIDS, tem como objetivo acabar com a epidemia de HIV até 2030, por meio de abordagens baseadas nos princípios dos direitos humanos, de respeito e inclusão (Unaids, 2020).

As variações anuais observadas na prevalência da infecção na população indígena do Polo Base, caracterizada por registros de quatro anos consecutivos com a identificação de zero casos pode estar associado a problemas relacionados à capacidade do serviço de saúde local e menos ao comportamento epidemiológico da infecção (Graeff et al., 2019) no entanto é preciso ter cautela, considerando a vulnerabilidade das populações indígenas às doenças infecciosas (Santos et al., 2020).

Estudos tem demonstrado o caráter endêmico da infecção pelo HIV entre as populações indígenas, com a preocupante possibilidade de se tornar uma epidemia em pouco tempo (Graeff et al., 2019). Agregando-se a isso temos os problemas históricos que acompanham os povos indígenas desde a colonização, sabendo-se a pobreza, o racismo, a marginalização, a falta de oportunidade para geração de renda entre outros, fatores importantes para a manutenção da invisibilidade desses povos para a sociedade geral (Ponce et al, 2017).

A aldeia Porto Lindo, que apresentou o maior número de casos identificados no Polo Base de Japorã, tem uma proximidade importante com a área urbana, além de fatores socioeconômicos que favorecem a saída de jovens e adultos em busca de trabalho fora do território indígena. Outro fator relevante é a proximidade com fronteiras entre países, uma vez que o território Guarani e Kaowá se estende para o Paraguai (Graeff et al., 2019), que favorece mobilidade expressiva destes indígenas entre o território nacional e internacional. Esses fatores aumentam a situação de vulnerabilidade, pois favorece a interiorização do HIV e da aids (Ribeiro et al., 2020).

É importante considerar que os indígenas vivendo com HIV apresentam necessidades diferentes com relação ao acompanhamento, sendo necessário incorporação dessas necessidades ao processo de cuidado. Sabe-se que as precárias condições sociais e econômicas dos pacientes indígenas fazem com que profissionais de saúde envolvidos no acompanhamento tentem suprir estas deficiências de forma que vivenciam sentimento de impotência diante dos desfechos desfavoráveis (Marx et al., 2020).

Sobre a taxa de mortalidade por HIV nestas populações observou-se que, apesar do acesso aos medicamentos antirretrovirais, existe descontinuidade do tratamento. A adesão ao tratamento corrobora para o aumento da qualidade de vida das pessoas vivendo com HIV, além de favorecer a expectativa de vida dessa população, reduzindo a carga viral a níveis indetectáveis (Zuge et al., 2017).

As taxas de mortalidade não se apresentam de forma equânime entre os polos base do DSEI-MS e se alteram de acordo com as especificidades locais, apontando para a necessidade de avaliação individual dos serviços de saúde, pois estes possuem forma peculiar de enfrentamento do problema, a partir da realidade local (Graeff et al., 2019).

Nota-se que os determinantes socioeconômicos, ambientais e culturais tornam a implementação de estratégias de tratamento e acompanhamento complexas (Graeff et al., 2019) e se esbarra na persistência de iniquidades em saúde que repercute no acesso às medidas de prevenção, ao diagnóstico precoce e à manutenção do tratamento, o que corrobora para a manutenção de altas taxas de prevalência e de mortalidade do HIV entre as populações indígenas (OPAS, 2003; Russel e al., 2019).

Embora seja identificado avanços no acompanhamento e tratamento de pessoas que vivem com HIV no contexto geral, nas populações indígenas, considerando a situação de vulnerabilidade, é fundamental reduzir as barreiras de acesso à 
saúde e melhorar os resultados de saúde por meio de uma abordagem intercultural de forma a garantir a promoção da saúde e prevenção da transmissão do HIV (Ruffinen et al., 2015; Russel et al., 2019). As inovações implementadas no ano 2000, tais como tratamento antiretrovial e profilaxia de pré exposição, fortaleceram as estratégias de enfrentamento da epidemia do HIV no âmbito do SUS. Essas ações se desenvolvem no sentido de responder localmente a estratégia global 90/90/90, que objetiva o fim da epidemia da aids até 2030 (Granich et al., 2017).

As ações de prevenção do HIV em populações indígenas configuram-se como importante desafio para a saúde pública do país, sendo necessário iniciativas que compreendam a complexidade das questões indígenas e o respeito às especificidades étnico-culturais desses povos de considerável vulnerabilidade socioambiental (Bastos et al., 2017).

\section{Conclusão}

A principal limitação do estudo está associada à qualidade das informações secundárias disponíveis nas fontes de dados utilizadas, o que implicou em ausência de registro, como o preenchimento do campo raça/cor no SINAN. No entanto, o banco fornecido pelas Secretarias Municipais de Saúde, foi previamente filtrado e disponibilizado exclusivamente para essa categoria de raça/cor e a utilização de uma segunda fonte de dados, própria da área técnica do DSEI-MS, permitiu rastrear um maior número de casos e, consequentemente, a cobertura da informação.

A despeito destas limitações, o presente estudo trouxe benefícios por abordar uma doença particularmente pouco conhecida na epidemiologia dos povos indígenas no Brasil, que traz impactos no perfil de morbimortalidade, e sua associação com as mudanças socioculturais resultantes do processo de interação com populações não indígenas. Os achados evidenciaram não somente o comportamento da doença, mas também a capacidade dos serviços em desenvolver ações de prevenção, de diagnóstico e tratamento precoce adequados.

Importante ainda reconhecer que a infecção se manifesta de forma diferente da população em geral e até mesmo entre os diferentes grupos de indígenas. Tendo em vista o perfil da população com maior vulnerabilidade para a infecção nos diferentes contextos étnicos, é necessário que abordagens para identificar as populações chaves entre os povos indígenas sejam realizadas de modo que ações direcionadas impactem os indicadores de prevalência da infecção.

Sugere-se ainda o desenvolvimento de mais pesquisas acerca da temática com um número amostral maior e considerando outras etnias, visto os benefícios que a compreensão deste fenômeno pode trazer para as populações indígenas.

\section{Referências}

Abati, P., \& Segurado, A. C. (2015). HIV testing and clinical status upon admission to a specialized health care unit in Pará, Brazil. Revista de Saude Publica, 49(1). https://doi.org/10.1590/S0034-8910.2015049004625

Bastos, J. L., Santos, R. V., Cruz, O. G., Longo, L. A. F. B., \& Da Silva, L. O. (2017). Características sociodemográficas de indígenas en los censos Brasileños de 2000 y 2010: Un enfoque comparativo. Cadernos de Saude Publica, 33, 1-17. https://doi.org/10.1590/0102-311X00085516

Benzaken, A. S., Meritxell, S., Brito, I., Bermúdez, N. S. B., Galbán, E., Peeling, R. W., et al. (2017). HIV and syphilis in the context of community vulnerability among indigenous people in the Brazilian Amazon. International Journal for Equity in Health, 16(1), 1-9. https://doi.org/10.1186/s12939-017$0589-8$

Brasil. Ministério da Saúde. (2000). Povos indígenas e a prevenção às DST, HIV e AIDS. 24.

Brasil. Ministério da Saúde. (2014). Portaria n ${ }^{0}$ 1.271, de 6 de junho de 2014. Define a Lista Nacional de Notificação Compulsória de doenças, agravos e eventos de saúde pública nos serviços de saúde públicos e privados em todo o território nacional, nos termos do anexo, e dá outras providências. Diário Oficial da União.

Brasil. Ministério da Saúde. (2017). Secretaria Especial de Atenção à Saúde Indígena. Caracterização demográfica, étnico-cultural dos povos indígenas do Distrito Sanitário Especial Indígena Mato Grosso do Sul. Brasília: Ministério da Saúde

Brasil. Ministério da Saúde. (2020). Boletim Epidemiológico HIV / Aids | 2020. Secretaria de Vigilância Em Saúde, 1, 68. http://www.aids.gov.br/ptbr/pub/2020/boletim-epidemiologico-hivaids-2020

Brasil. Ministério da Saúde. (2021). Boletim Epidemiológico HIV / Aids | 2021. Secretaria de Vigilância Em Saúde. 
Coimbra, A. E. A. (2014). Saúde e povos indígenas no Brasil: reflexões a partir do I Inquérito Nacional de Saúde e Nutrição Indígena. Cadernos de Saúde

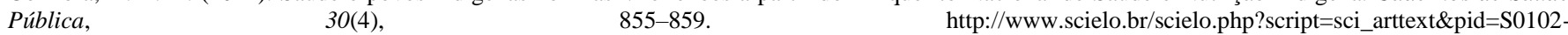
311X2014000400855\&lang=pt\%0Ahttp://www.scielo.br/pdf/csp/v30n4/0102-311X-csp-30-4-0855.pdf

Dartora, W. J., Ânflor, É. P., \& Silveira, L. R. P. (2017). HIV prevalence in Brazil 2005-2015: data from the Unified Health System Prevalencia. Revista Cuidarte, 1919-1928. http://dx.doi.org/10.15649/cuidarte.v8i3.462

Gomes, S. C., \& Esperidião, M. A. (2017). Indigenous peoples access to health services in Cuiabá, Mato Grosso State, Brazil. Cadernos de Saude Publica, 33(5). https://doi.org/10.1590/0102-311X00132215

Graeff, S. V., Pícolli, R. P., Arantes, R., Oliveira, V., Castro, L., \& Cunha, R. V. (2019). Aspectos epidemiológicos da infecção pelo HIV e da aids entre povos indígenas. Revista de Saúde Pública, 53:71. https://doi.org/10.11606/S1518-8787.2019053000362

Graeff, S. V.; Pícolli, R. P.; Arantes, R., \& Cunha, R. V. (2021). Evolution of HIV infection in indigenous peoples in Central Brazil. Cadernos de Saúde Pública, 37(12), 1-13. https://doi.org/10.1590/0102-311X000062920

Granich, R., Williams, B., Montaner, J., \& Zuniga, J. M. (2017). 90-90-90 and ending AIDS: necessary and feasible. The Lancet, 390(10092), 341-343. https://doi.org/10.1016/S0140-6736(17)31872-X

Granjeiro, A., Escuder, M. M. L., \& Castilho, E. A. (2010). A epidemia de AIDS no Brasil e as desigualdades regionais e de oferta de serviço. Cadernos de Saude Publica, 26(12), 2355-2367. https://doi.org/10.1590/S0102-311X2010001200014

IBGE. (2012). Os indígenas no Censo Demográfico 2010 primeiras considerações com base no quesito cor ou raça. Censo Demográfico 2010 , 232. https://ww2.ibge.gov.br/indigenas/indigena_censo2010.pdf\%0Ahttp://biblioteca.ibge.gov.br/visualizacao/livros/liv6687.pdf

Lima, I. B. (2018). Importância do diagnóstico precoce de hiv para a eficácia terapêutica e o bem-estar do paciente. CES Revista, 32(1), 57-71. https://seer.cesjf.br/index.php/cesRevista/article/view/1468/956

Maia, E. C. A., \& Reis-Junior, L. P. (2019). Modos de enfrentamento do HIV/AIDS: Direitos Humanos, vulnerabilidade e assistência. Rev. Nufen, 11(1), 178193. https://doi.org/10.26823/RevistadoNUFEN.vol11.nº1ensaio48

Marx, J., Acosta, L., Deschutter, E. J., Bornay, F. J., Sotilho, S. V., \& Rincon, J. M. R. (2020). Sífilis e infecção pelo HIV em indígenas Mbya Guarani em comunidades de Puerto IGuazu (Argentina): diagnóstico, contato, rastreamento e acompanhamento. Rev. Inst. Med. Trop. São Paulo. http://doi.org/10.1590/S1678-9946202062019

Maúngue, H. B. (2020). Mozambican woman: Culture, tradition and gender issues in the feminization of the HIV/ AIDS. Revista Estudos Feministas, 28(1), 1-13. https://doi.org/10.1590/1806-9584-2020V28N168328

Ministério da Saúde. (2020). Vulnerabilidades, impactos e o enfrentamento ao Covid-19 no contexto dos povos indígenas: reflexões para a ação. Fiocruz, 1-5. https://portal.fiocruz.br/observatorio-covid-19

Negin, J., Aspin, C., Gadsden, T., \& Reading, C. (2015). HIV Among Indigenous peoples: A Review of the Literature on HIV-Related Behaviour Since the Beginning of the Epidemic. AIDS and Behavior, 19(9), 1720-1734. https://doi.org/10.1007/s10461-015-1023-0

Nóbrega, R. G., Oliveira, N. A., Lima, É. A. R., Silva, A. C. O., Almeida, S. A., \& Nogueira, J. A. (2020). Prevenção do HIV sob o olhar de mulheres indígenas potiguaras. Revista de Enfermagem Da UFSM, 10, e64. https://doi.org/10.5902/2179769241396

Orellana, E. R., Alva, I. E., Cárcamo, C. P., \& Garcia, P. J. (2016). Structural Factors That Increase HIV/STI Vulnerability Among Indigenous People in the Peruvian Amazon. Physiology \& Behavior, 176(3), 139-148. https://doi.org/10.1177/1049732313502129.Structural

Organización Panamericana de La Salud P. (2003). Promoción de la salud sexual y prevención del VIH-sida y de las ITS en los pueblos indígenas de las Américas - Abya-Yala Kuyarinakui. OPAS, 41p.

Pereira, G. F. M., Pimenta, M. C., Giozza, S. P., Caruso, A. R., Bastos, F. I., \& Guimarães, M. D. C. (2019). HIV/AIDS, stis and viral hepatitis in Brazil: Epidemiological trends. Revista Brasileira de Epidemiologia, 22(Suppl 1), 1-2. https://doi.org/10.1590/1980-549720190001.supl.1

Ponce, P., Munõz, R., \& Stival, M. (2017). Pueblos indígenas, VIH y políticas públicas en Latinoamérica: Una exploración en el panorama actual de la prevalencia epidemiológica, la prevención, la atención y el seguimiento oportuno. Salud Colectiva, 13(3), 537-554. https://doi.org/10.18294/sc.2017.1120

Portocarrero, J. (2015). VIH en gestantes indígenas: un reto para la salud pública peruana. Revista Peruana de Medicina Experimental y Salud Pública, 32(3), 546. https://doi.org/10.17843/rpmesp.2015.323.1691

Polanco-Pasaje, J. E., Rodríguez-Márquez, I., Tello-Hoyos, K. Y., Torres-Pereda, P., Guzmán-Salazar, B. L., \& Pérez, F. (2021). Tuberculosis care cascade for the indigenous population in Colombia: an operational research study*. Revista Panamericana de Salud Publica/Pan American Journal of Public Health, 45, 1-9. https://doi.org/10.26633/RPSP.2021.20

Ribeiro, V. F., Alves, A. P. B., Argenta, L. B., \& Barreto, H. C. S. (2020). Estudo epidemiológico sobre o vírus da imunodeficiência humana (HIV) em indígenas do estado de Roraima entre 2010 a 2018. Revista Eletrônica Acervo Saúde, 12(7), e3447. https://doi.org/10.25248/reas.e3447.2020

Ruffinen, C. Z., Sabidó, M., Díaz-Bermúdez, X. P., Lacerda, M., Mabey, D., Peeling, R. W., et al. (2015). Point-of-care screening for syphilis and HIV in the borderlands: Challenges in implementation in the Brazilian Amazon. BMC Health Services Research, 15(1), 1-10. https://doi.org/10.1186/s12913-015-1155-y

Russel, N. K., Nazar, K., Pino, S., Gonzalez, M. A., Díaz-Bermúdez, X. P., \& Ravasi, G. (2019). HIV, syphilis, and viral hepatitis among Latin American indigenous peoples and Afro-descendants: A systematic review. Rev Panam Salud Publica, 43, 1-13. https://doi.org/10.26633/RPSP.2019.17

Santos, R. V., Guimarães, B. N., Campos, M. B., \& Azavedo, M. M. A. (2019). Entre Demografia e Antropologia: povos indígenas no Brasil. In Editora 
Research, Society and Development, v. 11, n. 3, e12711325985, 2022

(CC BY 4.0) | ISSN 2525-3409 | DOI: http://dx.doi.org/10.33448/rsd-v11i3.25985

FIOCRUZ, 2019, 264 p. Saúde dos povos Indígenas collection. https://doi.org/10.7476/9786557080139

Santos, N. J. S. (2016). To be black and woman: dual vulnerability to STD/HIV/AIDS. Saude e Sociedade, 25(3), 602-618. https://doi.org/10.1590/S0104129020162627

Santos, R. V., Coimbra, C. E. A., \& Radin, J. (2020). Why did they die?: Biomedical narratives of epidemics and mortality among amazonian indigenous populations in sociohistorical and anthropological contexts. Current Anthropology, 61(4), 441-470. https://doi.org/10.1086/710079

Schmitt, S. V., Andra, U. V., \& Chagas, A. C. F. (2018). Schmitt SV, et al HIV/Aids notifications in Mato Grosso do Sul. REvista Prevenção Em Saúde (REPIS), 1-8.

Silva, C. M., Alves, R. S., Santos, T. S., Bragagnollo, G. R.; Tavares, C. M., \& Santos, A. A. P. (2018). Epidemiological overview of HIV/AIDS in pregnant women from a state of northeastern Brazil. Revista Brasileira de Enfermagem, 71, 613-621. https://doi.org/http://dx.doi.org/10.1590/0034-7167-2017-0495

Teixeira, T. R. A., Gracie, R., Malta, M. S., \& Bastos, F. (2014). Social geography of AIDS in Brazil: identifying patterns of regional inequalities. Cad. Saúde Pública, 30(2), 259-271. http://www.scielosp.org/scielo.php?script=sci_arttext\&amp\%0Apid=S0102-311X2014000200259

Tiago, Z. S., Pícolli, R. P., Graeff, S. V., Cunha, R. V., \& Arantes, R. (2017). Underreporting of gestational, congenital and acquired syphilis among indigenous peoples in Mato Grosso do Sul State, Brazil, 2011-2014. Epidemiol. Serv. Saude, Brasília, 26(3), 26(3), 503-512. https://doi.org/10.5123/S167949742017000300008

Trindade, L. N. M., Nogueira, L. M. V., Gomes, E. S., Guimarães, J. T., Costa, N. Y., Santos, S. F. D., et al. (2021). Epidemiological overview of HIV in indigenous and non-indigenous pregnant women in the state of Para. Rev. Eletr. Enferm. [Internet]., 1-8. https://doi.org/10.5216/ree.v23.67563.

United Nations Joint Programme on HIV/AIDS (UNAIDS). (2020). UNAIDS Data 2020. Programme on HIV/AIDS, 1-248. http://www.unaids.org/sites/default/files/media_asset/20170720_Data_book_2017_en.pdf

United Nations Joint Programme on HIV/AIDS (UNAIDS). (2021). Report on the global AIDS epidemic 2021. Geneva: AIDS by the numbers.

Wozniak, R. J., Cerqueira, N. B., Dantas, M. C. S., Mahafe, B. ., Barros, D. A. C., Medeiros, E. A., et al. (2020). Factors associated with attitudes towards HIV cure research among transgender women and travestis: A cross-sectional survey in São Paulo, Brazil. BMJ Open, 10(11). https://doi.org/10.1136/bmjopen2020-040092

Zangirolami-Raimundo, J., Echeimberg, J. O., \& Leone, C. (2018). Tópicos de metodologia de pesquisa : Estudos de corte transversal. Journal of Human Growth and Development, v. 28, n. 3, p. 356-360.

Zuge, S. S., Primeira, M. R., Remor, E., Magnago, T. S. B. de S., Paula, C. C. de, \& Padoin, S. M. de M. (2017). Fatores associados à adesão ao tratamento antirretroviral em adultos infectados pelo HIV: estudo transversal. Revista de Enfermagem Da UFSM, 7(4), 577. https://doi.org/10.5902/2179769225657 